YITP-98-30, gr-qc/9805039

\title{
Comments on entanglement entropy
}

\author{
Shinji Mukohyama \\ Yukawa Institute for Theoretical Physics, Kyoto University \\ Kyoto 606-8502, Japan
}

(August 1, 2018)

\begin{abstract}
A new interpretation of entanglement entropy is proposed: entanglement entropy of a pure state with respect to a division of a Hilbert space into two subspaces 1 and 2 is an amount of information, which can be transmitted through 1 and 2 from a system interacting with 1 to another system interacting with 2. The transmission medium is quantum entanglement between 1 and 2. In order to support the interpretation, suggestive arguments are given: variational principles in entanglement thermodynamics and quantum teleportation. It is shown that a quantum state having maximal entanglement entropy plays an important role in quantum teleportation. Hence, the entanglement entropy is, in some sense, an index of efficiency of quantum teleportation. Finally, implications for the information loss problem and Hawking radiation are discussed.
\end{abstract}

PACS number(s): 04.70.Dy

Typeset using REVTEX 


\section{INTRODUCTION}

It is believed that the well-known analogy between the theory of black holes and thermodynamics, which is called black hole thermodynamics, is universal and that there should be a deep physics in its origin. In particular, since its first introduction by Bekenstein [1] as a quantity proportional to horizon area, black hole entropy has been one of the hottest topics in black hole physics. The black hole entropy is called the Bekenstein-Hawking entropy because the proportionality coefficient was determined to be $1 / 4$ by Hawking's discovery of thermal radiation from a black hole [2]. It is well known that the black hole entropy has various properties similar to those of thermodynamical entropy. For example, the sum of the black hole entropy and the entropy of matter outside the black hole does not decrease. This fact is called the generalized second law and was proved for a quasi-stationary black hole [3, 国. Moreover, the black hole entropy can be used to judge whether a black hole solution is stable or unstable [5]. In fact, it can be used as a potential function in catastrophe theory and results of the catastrophe theory coincide with those from a linear perturbation analysis. Hence, there are so many similarities between black hole entropy

and thermodynamical entropy that we expect that the former has a statistical origin, as the latter has.

Recently a microscopic derivation of the black hole entropy was given in superstring theory [6] by using the so-called D-brane technology [7]. In this approach the black hole entropy is identified with the logarithm of the number of states of massless strings attached to Dbranes, with D-brane configuration and total momentum of the strings along a compactified direction fixed to be consistent with the corresponding black hole [8]. The analysis along this line was extended to the so-called M-theory [9]. In particular, by using a conjectured correspondence (the Matrix theory) between the M-theory in the infinite momentum frame and a 10-dimensional $U(N)$ supersymmetric Yang-Mills theory dimensionally reduced to $(0+1)$-dimension with $N \rightarrow \infty$ [10], the black hole entropy was calculated by means of the Yang-Mills theory. The result gives the correct Bekenstein-Hawking entropy for BPS black 
holes and their low lying excitations [11]. Moreover, in Ref. [12] the black hole entropy of a Schwarzschild black hole was derived in the Matrix theory up to a constant of order 1 . On the other hand, in loop quantum gravity [13], black hole entropy was identified with the logarithm of the number of different spin-network states with the sum of eigenvalues of the area operator fixed [14]. The result coincides with the Bekenstein-Hawking entropy up to a constant of order 1 . It is evident that these derivations based on the candidate theories of quantum gravity depend strongly on details of the theories. In this sense, the success of the derivations can be considered as non-trivial consistency checks of the theories. However, it is believed that proportionality of the black hole entropy to horizon area is more universal and does not depend on details of the theory. Hence, one should be able to give a statistical or thermodynamical derivation of the black hole entropy, which does not depend on details of theory, while we are proceeding with theory-dependent derivations of it by using the well-established candidate theories of quantum gravity.

There were many attempts to explain the origin of the black hole entropy besides the above theory-dependent approaches. For example, in Euclidean gravity the black hole entropy is associated with the topology of an instanton which corresponds to a black hole [15] I; Wald [17] defined the black hole entropy as a Noether charge associated with a bifurcating Killing horizon f; 'tHooft [19] identified the black hole entropy with the statistical entropy of a thermal gas of quantum particles with a mirror-like boundary just outside the horizon (the brick wall model); Pretorius et al. [20] identified the black hole entropy with the thermodynamical entropy of a shell in thermal equilibrium with acceleration radiation due to the shell's gravity in the limit that the shell forms a black hole. There remains another strong candidate for the statistical origin of the black hole entropy, called entanglement

\footnotetext{
${ }^{1}$ The 1-loop correction to the black hole entropy was also calculated and compared with the brick wall model and the conical singularity method [16].

${ }^{2}$ Relations to the approach by Euclidean gravity was investigated in Ref. [18].
} 
entropy 21 23. It is a statistical entropy measuring the information loss due to a spatial division of a system [21]. The entanglement entropy is based only on the spatial division, and can be defined independently of the theory, although explicit calculations in the literature are dependent on the model employed. Moreover, as explained in the following argument, it is expected independently of the details of the theory that the entanglement entropy is proportional to the area of the boundary of the spatial division. In this sense, the entanglement entropy is considered to be a strong candidate for the statistical origin of the black hole entropy.

Now let us review the concept of the entanglement entropy. We consider a Hilbert space $\mathcal{F}$ constructed from two Hilbert spaces $\mathcal{F}_{1}$ and $\mathcal{F}_{2}$ as

$$
\mathcal{F}=\mathcal{F}_{1} \otimes \mathcal{F}_{2}
$$

From a pure density matrix $\rho=|\phi\rangle\langle\phi|$ on $\mathcal{F}$ we can define reduced density matrices $\rho_{1}$ and $\rho_{2}$ by $\rho_{1,2}=\operatorname{Tr}_{2,1} \rho$, where $\operatorname{Tr}_{1,2}$ represents a partial trace over $\mathcal{F}_{1,2}$, respectively, and $|\phi\rangle$ is an element of $\mathcal{F}$ with unit norm. The entanglement entropy is defined by $S_{\text {ent } 1}=S\left[\rho_{1}\right]$ or $S_{\text {ent } 2}=S\left[\rho_{2}\right]$, where $S[\cdot]$ is the von Neumann entropy. We can denote these two entropies by the same symbol $S_{\text {ent }}$ since $S_{\text {ent } 1}=S_{\text {ent } 2}$ (see Appendix of Ref. [24] for a proof).

In the context of black hole thermodynamics the entanglement entropy of matter fields on a black hole background is regarded as a strong candidate for black hole entropy. In this case the Hilbert space $\mathcal{F}$ is a space of all quantum states of matter fields and the direct product structure (11) of $\mathcal{F}$ is obtained from a division of a spacelike hypersurface into two regions: one inside and one outside of a boundary surface. Due to the above mentioned symmetric property $S_{\text {ent }}=S\left[\rho_{1}\right]=S\left[\rho_{2}\right]$, it is expected that $S_{\text {ent }}$ for a pure state is proportional to the area $A$ of the boundary. Thus,

$$
S_{\text {ent }}=c \frac{A}{a^{2}}
$$

is expected, where $c$ is a numerical constant of order 1 and $a$ is a cutoff length of the theory introduced in order to make the expression finite and dimensionless as entropy should be. In 
many references [21 23], this behavior of $S_{\text {ent }}$ was confirmed. So the entanglement entropy for a pure state has a property similar to black hole entropy, provided that the boundary is set to be close to a black hole horizon and the cutoff length is of Planck order. Moreover, in Ref. [25], it was shown that concepts of entanglement energy and entanglement temperature can be introduced for matter fields in a black hole background and that their behavior is the same as for the energy and temperature of the black hole. For these reasons the entanglement entropy has a potential to be the origin of the black hole entropy.

In Sec. II, based on a relation between the entanglement entropy and so-called conditional entropy, we propose an interpretation of the entanglement entropy. In Sec. III variational principles in entanglement thermodynamics are used to determine quantum states. In particular, a state having maximal entanglement entropy is determined and is used in Sec. IV to transmit information about an unknown quantum state. Sec. $\nabla$ is devoted to a summary of this paper and to discuss implications for the information loss problem and Hawking radiation.

\section{CONDITIONAL ENTROPY AND ENTANGLEMENT ENTROPY}

Entropy plays important roles not only in statistical mechanics but also in information theory. In the latter, entropy of a random experiment, each of whose outcomes has an attached probability, represents uncertainty about the outcome before performing the experiment [28]. Besides the well-known Shannon entropy, there exist various definitions of entropies in information theory. For example, the so-called conditional entropy of an experiment $A$ on another experiment $B$ is defined by $H(A \mid B)=-\sum_{a, b} p(a, b) \ln p(a \mid b)$, where $a$ and $b$ represent outcomes of $A$ and $B$, respectively, $p(a, b)$ is a joint probability of $a$ and $b$, and $p(a \mid b)=p(a, b) / p(b)$ is a conditional probability of $a$ on $b$. Here $p(b)$ is a probability of $b$. The conditional entropy corresponds to an uncertainty about the outcome of $A$ after the experiment $B$ is done. In other words it can be regarded as the amount of information about $A$ which cannot be known from the experiment $B$. The quantum analogue of the 
conditional entropy was considered in references [26,27] and is called the von Neumann conditional entropy. Consider a Hilbert space $\mathcal{F}$ of the form (11) and let $\rho$ be a density matrix on $\mathcal{F}$. The von Neumann conditional entropy of $\rho$ about the subsystem 1 on the subsystem 2 is defined by

$$
S_{1 \mid 2}=\operatorname{Tr}\left[\rho \sigma_{1 \mid 2}\right]
$$

where $\sigma_{1 \mid 2}=\mathbf{1}_{1} \otimes \ln \rho_{2}-\ln \rho$. The von Neumann conditional entropy $S_{2 \mid 1}$ of $\rho$ about the subsystem 2 on the subsystem 1 is defined in a similar way. It is expected that $S_{1 \mid 2}$ (or $S_{2 \mid 1}$ ) represents the amount of the information about the subsystem 1 (or 2) which cannot be known from 2 (or 1 , respectively).

The von Neumann conditional entropy can be negative. In fact, it is easy to see that

$$
S_{1 \mid 2}=S_{2 \mid 1}=-S_{\text {ent }},
$$

if $\rho$ is a pure state. Hence, if $\rho$ is a pure state then the conditional entropy is zero or negative. Our question now is "what is the meaning of the negative conditional entropy of a pure state?' It might be expected that $\left|S_{1 \mid 2}\right|\left(=S_{\text {ent }}\right)$ is the amount of the information about 1 (or 2) which can be known from 2 (or 1, respectively). However, this statement is not precise. A precise statement is that it is an amount of information, which can be transmitted through 1 and 2 from a system interacting with 1 to another system interacting with 2. The transmission medium is quantum entanglement between 1 and 2 .

The purpose of the remaining part of this paper is to give suggestive arguments for this statement.

\section{VARIATIONAL PRINCIPLES IN ENTANGLEMENT THERMODYNAMICS}

In statistical mechanics, the von Neumann entropy is used to determine an equilibrium state: an equilibrium state of an isolated system is determined by maximizing the entropy. Thus, we expect that the entanglement entropy may be used to determine a quantum state. 
As an illustration we consider a simple system of two particles, each with spin $1 / 2$ : we consider a Hilbert space $\mathcal{F}$ of the form (11) and denote an orthonormal basis of $\mathcal{F}_{i}$ by $\left\{|\uparrow\rangle_{i},|\downarrow\rangle_{i}\right\}(i=1,2)$. Let $|\phi\rangle$ be an element of $\mathcal{F}$ with unit norm and expand it as

$$
|\phi\rangle=a|\uparrow\rangle_{1} \otimes|\uparrow\rangle_{2}+b|\uparrow\rangle_{1} \otimes|\downarrow\rangle_{2}+c|\downarrow\rangle_{1} \otimes|\uparrow\rangle_{2}+d|\downarrow\rangle_{1} \otimes|\downarrow\rangle_{2},
$$

where $|a|^{2}+|b|^{2}+|c|^{2}+|d|^{2}=1$ is understood. The corresponding reduced density matrix is given by

$$
\begin{aligned}
\rho_{2}= & \left(|a|^{2}+|c|^{2}\right)|\uparrow\rangle_{22}\left\langle\uparrow\left|+\left(a b^{*}+c d^{*}\right)\right| \uparrow\right\rangle_{22}\langle\downarrow| \\
& +\left(a^{*} b+c^{*} d\right)|\downarrow\rangle_{22}\left\langle\uparrow\left|+\left(|b|^{2}+|d|^{2}\right)\right| \downarrow\right\rangle_{22}\langle\downarrow|
\end{aligned}
$$

and the entanglement entropy can be easily calculated from it. The resulting expression for the entanglement entropy is

$$
S_{e n t}=-\frac{1+x}{2} \ln \left(\frac{1+x}{2}\right)-\frac{1-x}{2} \ln \left(\frac{1-x}{2}\right),
$$

where $x=\sqrt{1-4|a d-b c|^{2}}$. By requiring $d S_{\text {ent }} / d x=0$ we obtain the condition $|a d-b c|=$ $1 / 2$. Thus a state maximizing the entanglement entropy is

$$
|\phi\rangle=\frac{1}{\sqrt{2}}\left(|\uparrow\rangle_{1} \otimes|\downarrow\rangle_{2}-|\downarrow\rangle_{1} \otimes|\uparrow\rangle_{2}\right)
$$

up to a unitary transformation in $\mathcal{F}_{1}$ and the corresponding maximal value of the entanglement entropy is $\ln 2$. This state is well known as the EPR state.

It is notable that the corresponding reduced density matrix $\rho_{2}$ represents the microcanonical ensemble. This fact is related to the fact that the maximum of entropy gives the microcanonical ensemble in statistical mechanics. Thus, in general, if $\mathcal{F}_{1}$ and $\mathcal{F}_{2}$ have the same finite dimension $N$ then a state maximizing the entanglement entropy is written as

$$
|\phi\rangle=\frac{1}{\sqrt{N}} \sum_{n=1}^{N}\left(|n\rangle_{1} \otimes|n\rangle_{2}\right)
$$

up to a unitary transformation in $\mathcal{F}_{1}$, where $|n\rangle_{1}$ and $|n\rangle_{2}(n=1,2, \cdots, N)$ are orthonormal basis of $\mathcal{F}_{1}$ and $\mathcal{F}_{2}$, respectively. (See Appendix $\mathbb{A}$ for a systematic derivation. ) In the next section we use the state (9) to transmit information about an unknown quantum state. 
In statistical mechanics, free energy $F=E-T S$ can also be used to determine a statistical state: its minimum corresponds to an equilibrium state of a subsystem in contact with a heat bath of temperature $T$, provided that $T$ is fixed. This variational principle in statistical mechanics is based on the following three assumptions.

1. The total system (the subsystem + the heat bath) obeys the principle of maximum of entropy.

2. Total energy (energy of the subsystem + energy of the heat bath) is conserved.

3. The 1st law of thermodynamics holds for the heat bath.

Is there a corresponding variational principle in the quantum system in the Hilbert space $\mathcal{F}$ of the form (11)? The answer is yes. In Ref. [24] and Ref. [25] a concept of entanglement energy was introduced and a thermodynamical structure, which we call entanglement thermodynamics, was constructed by using the entanglement entropy and the entanglement energy. Thus we expect that entanglement free energy $F_{\text {ent }}$ defined as follows plays an important role in entanglement thermodynamics.

$$
F_{\text {ent }}=E_{\text {ent }}-T_{\text {ent }} S_{\text {ent }},
$$

where $E_{\text {ent }}$ is the entanglement energy and $T_{\text {ent }}$ is a constant. Among several options, we adopt the following definition of the entanglement energy.

$$
E_{\text {ent }}=\operatorname{Tr}\left[\rho: H_{2}:\right]
$$

where $\mathrm{H}_{2}$ is Hamiltonian of the subsystem 2, and : - : represents the normal ordering [25].

As shown in the following arguments, by minimizing the entanglement free energy, we can obtain a state in $\mathcal{F}$ characterized by the constant $T_{\text {ent }}$. Before doing it, here we consider a physical meaning of the principle of minimum of the entanglement free energy. Let us introduce another Hilbert space $\mathcal{F}_{\text {bath }}$, which plays the role of the heat bath in the above statistical-mechanical consideration, and decompose it to the direct product $\mathcal{F}_{\text {bath }}=\mathcal{F}_{\text {bath } 1} \otimes$ 
$\mathcal{F}_{\text {bath2. }}$. In this situation it is expected that the principle of the minimum entanglement free energy corresponds to the following situation.

1. The total system $\mathcal{F}_{\text {tot }} \equiv \mathcal{F} \otimes \mathcal{F}_{\text {bath }}$ obeys the principle of maximum of the entanglement entropy with respect to the decomposition $\mathcal{F}_{\text {tot }}=\mathcal{F}_{\text {tot } 1} \otimes \mathcal{F}_{\text {tot } 2}$, where $\mathcal{F}_{\text {tot } 1} \equiv \mathcal{F}_{1} \otimes \mathcal{F}_{\text {bath } 1}$ and $\mathcal{F}_{\text {tot } 2} \equiv \mathcal{F}_{2} \otimes \mathcal{F}_{\text {bath } 2}$.

2. Total entanglement energy (entanglement energy for $\mathcal{F}+$ entanglement energy for $\left.\mathcal{F}_{\text {bath }}\right)$ is conserved.

3. The 1st law of entanglement thermodynamics [24]

$$
d E_{\text {ent }}=T_{\text {ent }} d S_{\text {ent }}
$$

holds for $\mathcal{F}_{\text {bath }}$. In this situation we call the constant $T_{\text {ent }}$ the entanglement temperature.

It must be mentioned here that the variational principle of minimum of the entanglement free energy is not as fundamental as the principle of maximum of the entanglement entropy but is an approximation to the latter principle for a large system. However, like the principle of minimum free energy in statistical mechanics, the former principle should be a very useful tool to determine a quantum state.

We now calculate $F_{\text {ent }}$ for the system of two spin- $1 / 2$ particles and minimize it. For simplicity we adopt the following Hamiltonian for the subsystem 2:

$$
\begin{aligned}
& { }_{2}\left\langle\uparrow\left|: H_{2}:\right| \uparrow\right\rangle_{2}=\epsilon, \\
& { }_{2}\left\langle\uparrow\left|: H_{2}:\right| \downarrow\right\rangle_{2}=0, \\
& { }_{2}\left\langle\downarrow\left|: H_{2}:\right| \downarrow\right\rangle_{2}=0,
\end{aligned}
$$

where $\epsilon$ is a positive constant. The entanglement free energy $F_{\text {ent }}$ for the state (5) is given by

$$
F_{\text {ent }}=\epsilon\left(|a|^{2}+|c|^{2}\right)+T_{\text {ent }}\left[\frac{1+x}{2} \ln \left(\frac{1+x}{2}\right)+\frac{1-x}{2} \ln \left(\frac{1-x}{2}\right)\right] .
$$


By minimizing it we obtain the following expression for the state $|\phi\rangle$ up to a unitary transformation in $\mathcal{F}_{1}$.

$$
|\phi\rangle=\frac{1}{\sqrt{Z}}\left[e^{-\epsilon / 2 T_{\text {ent }}}|\uparrow\rangle_{1} \otimes|\uparrow\rangle_{2}+|\downarrow\rangle_{1} \otimes|\downarrow\rangle_{2}\right]
$$

where $Z=e^{-\epsilon / T_{\text {ent }}}+1$.

The corresponding reduced density matrix $\rho_{2}$ on $\mathcal{F}_{2}$ represents a canonical ensemble with temperature $T_{\text {ent }}$. This fact is related to the fact that the principle of minimum of free energy results in a canonical ensemble in statistical mechanics. Thus, in general, if $\mathcal{F}_{1}$ and $\mathcal{F}_{2}$ have the same finite dimension $N$ then a state minimizing the entanglement free energy is written as

$$
|\phi\rangle=\frac{1}{\sqrt{Z}} \sum_{n=1}^{N}\left[e^{-E_{n} / 2 T_{e n t}}|n\rangle_{1} \otimes|n\rangle_{2}\right]
$$

up to a unitary transformation in $\mathcal{F}_{1}$, where $Z=\sum_{n=1}^{N} e^{-E_{n} / T_{e n t}}$, and $E_{n}$ and $|n\rangle_{2}$ $(n=1,2, \cdots, N)$ are eigenvalues and orthonormalized eigenstates of the normal-ordered Hamiltonian of the subsystem 2. (See Appendix A for a systematic derivation.)

The state (16) can be obtained also from another version of the principle of maximum of the entanglement entropy: if we maximize $S_{\text {ent }}$ with $E_{\text {ent }}$ fixed then the state (16) is obtained. In this case, the constant $T_{\text {ent }}$ is determined so that the entanglement energy coincides with the fixed value.

Note that in Eq. (16) the infinite-dimensional limit $N \rightarrow \infty$ can be taken, provided that $T_{\text {ent }}$ is bounded. In this limit, the state (16) has the same form as those appearing in the thermo field dynamics of black holes [32] and the quantum field theory on a collapsing star background [33]. In fact, if we can set the value of the entanglement temperature of $\mathcal{F}_{\text {bath }}$ to be the black hole temperature then the state (16) in the limit completely coincides with those in Ref. [32,33]. In Ref. [25] it was shown numerically that the entanglement temperature for a real massless scalar field in a Schwarzschild spacetime is finite and equal to the black hole temperature of the background geometry up to a numerical constant of order 1. The finiteness of the entanglement temperature in the Schwarzschild spacetime is a 
result of cancellation of divergences in entanglement entropy and entanglement energy [25]. Thus, the finiteness is preserved even in the limit of zero cutoff length $(a \rightarrow 0)$.

\section{QUANTUM TELEPORTATION}

In Ref. [29] Bennet et al. proposed a method of teleportation of an unknown quantum state from one place to another. It is called quantum teleportation. In their method the information about the quantum state is separated into a 'quantum channel' and a 'classical channel', and each channel is sent separately from a sender "Alice" to a receiver "Bob". What is important is that the quantum channel is sent in a superluminal way by using a quantum correlation or entanglement, while the classical channel is transmitted at most in the speed of light. Here we mention that causality is not violated in an informational sense since Bob cannot obtain any useful information about the unknown state before the arrival of the classical channel. Hence Alice has to deliver the classical channel to Bob without fail. On the contrary she does not need to worry about whether the information in the quantum channel arrives at Bob's hand since the arrival is guaranteed by the quantum mechanics. It is notable that recently quantum teleportation was confirmed by experiments [30,31].

In this section we generalize the arguments in Ref. [29] to more abundant situations and try to reformulate it in terms of the entanglement entropy.

Let us consider a Hilbert space $\mathcal{F}$ of the form (1) with $\mathcal{F}_{i}$ constructed from Hilbert spaces $\mathcal{F}_{i \pm}$ as

$$
\mathcal{F}_{i}=\mathcal{F}_{i+} \otimes \mathcal{F}_{i-} .
$$

For example, consider matter fields in a black hole spacetime formed by gravitational collapse. In this situation, let $\mathcal{H}_{1}$ be a space of all wave packets on the future event horizon and $\mathcal{H}_{2}$ be a space of all wave packets on the future null infinity, and decompose each $\mathcal{H}_{i}$ into a high frequency part $\mathcal{H}_{i+}$ and a low frequency part $\mathcal{H}_{i-}$. Typically, we suppose the decomposition at an energy scale of Planck order. If we define $\mathcal{F}_{i \pm}$ as Fock spaces constructed from 
$\mathcal{H}_{i \pm}$, respectively, then the space $\mathcal{F}$ of all quantum states of the matter fields is given by (1) with (17). Although the following arguments do not depend on the construction of the Hilbert space $\mathcal{F}$, this example should be helpful for us to understand the physical meaning of the results obtained.

For simplicity we consider the case that all $\mathcal{F}_{i \pm}$ have the same finite dimension $N$ although in the above example of field theory the dimensions of the Hilbert spaces are infinite ${ }^{3}$. The main purpose of this section is to see general properties of the entanglement entropy by using a finite system. Anyway, the above example of field theory may be helpful to understand the following arguments. It will be investigated elsewhere how to generalize the arguments of quantum teleportation to infinite dimensions. In the finite dimensional case we assume the following three physical principles.

(a) A quantum state $|\phi\rangle$ in $\mathcal{F}$ is a direct product state given by $\left|\phi_{+}\right\rangle_{+} \otimes\left|\phi_{-}\right\rangle_{-}$, where $\left|\phi_{ \pm}\right\rangle_{ \pm}$are elements of $\mathcal{F}_{ \pm}=\mathcal{F}_{1 \pm} \otimes \mathcal{F}_{2 \pm}$, respectively.

(b) $\left|\phi_{+}\right\rangle_{+}$is determined by the principle of maximum of the entanglement entropy with respect to the decomposition $\mathcal{F}_{+}=\mathcal{F}_{1+} \otimes \mathcal{F}_{2+}$.

(c) A complete measurement of the von Neumann type on the joint system $\mathcal{F}_{1}$ is performed by a sender (Alice) in the orthonormal basis $\left\{\left|\psi_{n m}\right\rangle_{1}\right\}$, each of which maximizes the entanglement entropy with respect to the decomposition $\mathcal{F}_{1}=\mathcal{F}_{1+} \otimes \mathcal{F}_{1-}$.

In other words the assumption (c) is stated as follows: the state $|\phi\rangle$ is projected by one of

\footnotetext{
${ }^{3}$ In applying the results for finite dimensions to field theory, we have to introduce a regularization scheme to make the system finite. For example, we can discretize the system by introducing a cutoff length. After that, we can consider a finite dimensional subspace of the total Hilbert space of the discretized theory, for example, by restricting total energy to be less than the mass of the background geometry. After performing all calculations, we have to confirm that the infinitedimensional limit can be taken. See, for example, the final paragraph of the previous section.
} 
the basis $\left|\psi_{n m}\right\rangle_{1}$.

In the following arguments, under these assumptions, we show a possibility of quantum teleportation of the state $\left|\phi_{-}\right\rangle_{-}$in $\mathcal{F}_{-}$to $\mathcal{F}_{2}$ : we make a clone of $\left|\phi_{-}\right\rangle_{-}$by using the quantum entanglement which the state $\left|\phi_{+}\right\rangle_{+}$has. Therefore a receiver (Bob), who cannot contact with $\mathcal{F}_{1}$, may be able to get all information about the state $\left|\phi_{-}\right\rangle_{-}$in $\mathcal{F}_{-}$, provided that he can manage to get the classical channel.

Now let us show that explicitly. By the assumption (b) and the arguments in Sec. 【II (see Eq. (9)), the state $\left|\phi_{+}\right\rangle_{+}$can be written as

$$
\left|\phi_{+}\right\rangle_{+}=\frac{1}{\sqrt{N}} \sum_{n=1}^{N}|n\rangle_{1+} \otimes|n\rangle_{2+},
$$

where $\left\{|n\rangle_{i+}\right\}(n=1,2, \cdots, N)$ are an orthonormal basis of $\mathcal{F}_{i+}$. Next, expand $\left|\phi_{-}\right\rangle_{-}$as

$$
\left|\phi_{-}\right\rangle_{-}=\sum_{n m} C_{n m}|n\rangle_{1-} \otimes|m\rangle_{2-}
$$

where $\left\{|n\rangle_{i-}\right\}(n=1,2, \cdots, N)$ are an orthonormal basis of $\mathcal{F}_{i-}$, and $\sum_{n m}\left|C_{n m}\right|^{2}=1$ is understood. To impose the assumption (c), we adopt the following basis $\left\{\left|\psi_{n m}\right\rangle_{1}\right\}(n, m=$ $1,2, \cdots, N)$, each of which maximizes the entanglement entropy.

$$
\left|\psi_{n m}\right\rangle_{1}=\frac{1}{\sqrt{N}} \sum_{j=1}^{N} e^{2 \pi i j n / N}|(j+m) \bmod N\rangle_{1+} \otimes|j\rangle_{1-} .
$$

In Appendix B it is proved that (20) is unique up to a unitary transformation in $\mathcal{F}_{1+}$. Hence, $|\phi\rangle=\left|\phi_{+}\right\rangle_{+} \otimes\left|\phi_{-}\right\rangle_{-}$is written as

$$
|\phi\rangle=\frac{1}{N} \sum_{n m}\left|\psi_{n m}\right\rangle_{1} \otimes U_{n m}^{(2+)}\left|\tilde{\phi}_{2}\right\rangle_{2}
$$

where $\left|\tilde{\phi}_{2}\right\rangle_{2}$ is a state in $\mathcal{F}_{2}$ given by

$$
\left|\tilde{\phi}_{2}\right\rangle_{2}=\sum_{n^{\prime} m^{\prime}} C_{n^{\prime} m^{\prime}}\left|n^{\prime}\right\rangle_{2+} \otimes\left|m^{\prime}\right\rangle_{2-}
$$

and $U_{n m}^{(2+)}(n, m=1,2, \cdots, N)$ are unitary transformations in $\mathcal{F}_{2+}$ defined by

$$
U_{n m}^{(2+)}=\sum_{k=1}^{N} e^{-2 \pi i k n / N}|(k+m) \bmod N\rangle_{2+2+}\langle k| .
$$


(See Appendix B for an explicit derivation of (21).)

Thus, after the measurements in the basis $\left\{\left|\psi_{n m}\right\rangle_{1}\right\}$ by the sender (Alice), the original state $|\phi\rangle$ jumps to one of the states $\left|\tilde{\phi}_{n m}\right\rangle$ defined by

$$
\left|\tilde{\phi}_{n m}\right\rangle=\left|\psi_{n m}\right\rangle_{1} \otimes U_{n m}^{(2+)}\left|\tilde{\phi}_{2}\right\rangle_{2}
$$

This state can be seen by the receiver (Bob), who cannot contact with $\mathcal{F}_{1}$, as the state $U_{n m}^{(2+)}\left|\tilde{\phi}_{2}\right\rangle_{2}$ in $\mathcal{F}_{2}$. Here note that the unitary transformation $U_{n m}^{(2+)}$ in $\mathcal{F}_{2+}$ is completely determined by a pair of integers $n$ and $m$ (outcome of the experiment by Alice). Thus, if the two integers are sent to the receiver (Bob) in the classical channel, then by operating the inverse transformation of the corresponding unitary transformation in $\mathcal{F}_{2+}$ the receiver (Bob) can obtain the 'clone' state $\left|\tilde{\phi}_{2}\right\rangle_{2}\left(\in \mathcal{F}_{2}\right)$ of $\left|\phi_{-}\right\rangle\left(\in \mathcal{F}_{-}\right)$. It is evident that $\left|\tilde{\phi}_{2}\right\rangle_{2}$ has all information about the original state $\left|\phi_{-}\right\rangle$.

It is remarkable that information to be sent to the receiver (Bob) in the classical channel is only two integers $n$ and $m$, while information included in the unknown state $\left|\phi_{-}\right\rangle_{-}$is a set of complex constants $\left\{C_{n m}\right\}(n, m=1,2, \cdots, N)$ with a constraint $\sum_{n m}\left|C_{n m}\right|^{2}=1$. Thus a large amount of information is sent in the quantum channel. Here we mention that tracing out $\mathcal{F}_{2+}$ from the state $U_{n m}^{(2+)}\left|\tilde{\phi}_{2}\right\rangle_{2}$ or $\left|\tilde{\phi}_{2}\right\rangle_{2}$ results in the following density matrix $\rho_{2-}$ on $\mathcal{F}_{2-}:$

$$
\rho_{2-}=\sum_{n m}\left(\sum_{j} C_{j n} C_{j m}^{*}\right)|n\rangle_{2-2-}\langle m|,
$$

which is equivalent to the density matrix obtained by tracing out $\mathcal{F}_{1-}$ from the original unknown state $\left|\phi_{-}\right\rangle_{-}$. Hence, if the receiver (Bob) cannot contact with $\mathcal{F}_{2+}$, he does not obtain any information from the sender (Alice).

Finally it must be mentioned that the success of quantum teleportation is due to the state $\left|\phi_{+}\right\rangle_{+}$which has maximal entanglement entropy. If we took $\left|\phi_{+}\right\rangle_{+}$with less entanglement entropy then the teleportation would be less successful. Therefore, the entanglement entropy can be regarded as an index of efficiency of quantum teleportation. This consideration supports the interpretation of the entanglement entropy proposed in Sec. II. 


\section{SUMMARY AND DISCUSSION}

In this paper a new interpretation of entanglement entropy has been proposed based on its relation to the so-called conditional entropy and a well-known meaning of the latter. It is conjectured that entanglement entropy of a pure state with respect to a division of a Hilbert space into two subspaces 1 and 2 is an amount of information, which can be transmitted through 1 and 2 from a system interacting with 1 to another system interacting with 2 . The medium of the transmission is quantum entanglement between 1 and 2 .

To support the interpretation we have given the following two suggestive arguments: variational principles in entanglement thermodynamics and quantum teleportation. The most important variational principle we considered is the principle of maximum of entanglement entropy. This principle determines a state uniquely up to a unitary transformation in one of the two Hilbert subspaces (not in the whole Hilbert space). From the proposed conjecture it is expected that information can be transmitted most effectively through the two subspaces by using the maximal entanglement of the state. In fact, reformulating the quantum teleportation in terms of the entanglement entropy, we have shown that the state having maximal entanglement entropy plays an important role in quantum teleportation. This consideration gives strong support to our interpretation.

It is confirmed in many references [21 23] that the entanglement entropy has the same value as the black hole entropy up to a numerical constant of order 1 , provided that a cutoff length of Planck order is introduced in the theory. Hence we have a large amount of entanglement entropy to transmit information from inside to outside of a black hole by using quantum entanglement.

Hence, in our interpretation, it seems that the entanglement entropy is a quantity which cancels the black hole entropy to restore information loss, provided that the black hole entropy represents the amount of the information loss. For example, suppose that a black hole is formed from an initial state with zero entropy $(S=0)$. In this case, non-zero black hole entropy is generated $\left(S_{B H}>0\right)$ from the zero entropy state. At the same 
time, entanglement entropy and negative conditional entropy are also generated and their absolute values are as large as the black hole entropy $\left(S_{\text {ent }}=\left|S_{\text {cond }}\right| \simeq S_{B H}\right)$. After that, the black hole evolves by emitting Hawking radiation, changing the value of $S_{B H}$ and $S_{\text {ent }}$ (=|S $\left.S_{\text {cond }} \mid\right)$ with $S_{B H} \simeq S_{\text {ent }}$ kept. Finally, when the black hole evaporates, the entanglement entropy cancels the black hole entropy to settle the final entropy to be zero $(S=0)$. To summarize, the black hole entropy is an amount of temporarily missing information and the entanglement entropy is a quantity which cancels the black hole entropy. Both entropies appear and disappear together from the sea of zero entropy state.

As a by-product we have shown that the variational principle of minimum of entanglement free energy is useful to determine a quantum state. The resulting quantum state has exactly the same form as those appearing in the thermo field dynamics of black holes [32] and the quantum field theory on a collapsing black hole background [33], provided that the entanglement temperature $T_{\text {ent }}$ is set to be the black hole temperature. It is remarkable that $T_{\text {ent }}$ for a real massless scalar field in a Schwarzschild spacetime is equal to the black hole temperature of the background geometry up to a numerical constant of order 1 [25]. Thus we can say that the variational principle of minimum of entanglement free energy gives a new derivation of the Hawking radiation. Finally, we mention that with this variational principle the entanglement thermodynamics is equivalent to 'tHooft's brick wall model [19].

It will be valuable to analyze how to generalize arguments in this paper to the situation that divergences in entropy and energy are absorbed by renormalization [35,36]. If the generalization is achieved, the physical meaning of the entanglement entropy in black hole physics will become clearer. It is noteworthy that in the brick wall model the divergence in thermal energy is exactly canceled by divergence in negative energy [37].

Now the final comment is in order. It is worthwhile to clarify in what physical situations the variational principles can be applicable. (In thermodynamics the second law supports the principle of maximum entropy.) In other words, in what situations does the entanglement entropy increase? In what situations does the entanglement free energy decrease? To answer these questions, theorem 2 of Ref. [4] or its generalization may be useful. 


\section{Acknowledgments}

The author thanks Professor W. Israel for helpful discussions and so many stimulating suggestions. Without his help, this work could not be done. The author is grateful to S. A. Hayward for a careful reading of the manuscript and to Professor H. Kodama for continuous encouragement. The author was supported by JSPS Research Fellowships for Young Scientists, and this work was supported partially by the Grand-in-Aid for Scientific Research Fund (No. 9809228).

\section{APPENDIX A:}

In this appendix we give derivations of formulas (9) and (16).

We consider a Hilbert space $\mathcal{F}$ of the form

$$
\mathcal{F}=\mathcal{F}_{1} \otimes \mathcal{F}_{2}
$$

where $\mathcal{F}_{1}$ and $\mathcal{F}_{2}$ are Hilbert spaces with the same finite dimension $N$. An arbitrary unit element $|\phi\rangle$ of $\mathcal{F}$ is decomposed as

$$
|\phi\rangle=\sum_{n=1}^{N} \sum_{m=1}^{N} C_{n m}|n\rangle_{1} \otimes|m\rangle_{2},
$$

where $|n\rangle_{1}$ and $|n\rangle_{2}(n=1,2, \cdots, N)$ are orthonormal bases of $\mathcal{F}_{1}$ and $\mathcal{F}_{2}$, respectively, and $\sum_{n, m}\left|C_{n m}\right|^{2}=1$ is understood. Here, without loss of generality, we can choose the orthonormal basis of $\mathcal{F}_{2}$ to be eigenstates of the normal-ordered Hamiltonian : $H_{2}$ : of the sub-system as

$$
: H_{2}:|n\rangle_{2}=E_{n}|n\rangle_{2}
$$

Since $C^{\dagger} C$ is a non-negative hermitian matrix, we can define a set of non-negative real numbers $\left\{p_{n}\right\}$, each of which is the eigenvalue of the matrix $C^{\dagger} C$. Hence,

$$
C^{\dagger} C=V^{\dagger} P V
$$


where $V$ is a unitary matrix and $P$ is a diagonal matrix with diagonal elements $\left\{p_{n}\right\}$. With these definitions, the entanglement entropy $S_{\text {ent }}$ and the entanglement free energy $F_{\text {ent }}$ are calculated as

$$
\begin{aligned}
& S_{\text {ent }}=-\sum_{n=1}^{N} p_{n} \ln p_{n}, \\
& F_{\text {ent }}=\sum_{n=1}^{N} \sum_{m=1}^{N} E_{n} p_{m}\left|V_{m n}\right|^{2}+T_{\text {ent }} \sum_{n=1}^{N} p_{n} \ln p_{n} .
\end{aligned}
$$

The constraints $\sum_{n, m}\left|C_{n m}\right|^{2}=1$ and $V^{\dagger} V=\mathbf{1}$ are equivalent to

$$
\begin{aligned}
\sum_{n=1}^{N} p_{n} & =1, \\
\sum_{l=1}^{N} V_{l n}^{*} V_{l m} & =\delta_{n m} .
\end{aligned}
$$

Thus, the variational principles are restated as follows: to maximize (A5) under the constraints (A7); to minimize (A6) under the constraints (A7).

Now, we shall show that expressions (A5) and (A6) are same as those for entropy and free energy in statistical mechanics in the subspace $\mathcal{F}_{2}$. Let us consider a density operator $\bar{\rho}$ on $\mathcal{F}_{2}$ :

$$
\bar{\rho}=\sum_{n=1}^{N} \sum_{m=1}^{N} \tilde{P}_{n m}|n\rangle_{22}\langle m|,
$$

where $\tilde{P}_{n m}$ is an $N \times N$ non-negative hermitian matrix with unit trace. By diagonalizing the matrix $\tilde{P}$ as

$$
\tilde{P}=\bar{V}^{\dagger} \bar{P} \bar{V}
$$

we obtain the following expressions for entropy and free energy.

$$
\begin{aligned}
& S=-\sum_{n=1}^{N} \bar{p}_{n} \ln \bar{p}_{n} \\
& F=\sum_{n=1}^{N} \sum_{m=1}^{N} E_{n} \bar{p}_{m}\left|V_{m n}\right|^{2}+T \sum_{n=1}^{N} \bar{p}_{n} \ln \bar{p}_{n}
\end{aligned}
$$

where $\left\{\bar{p}_{n}\right\}$ are diagonal elements of the matrix $\bar{P}$ and $T$ is temperature. The constraints $\operatorname{Tr} \bar{\rho}=\mathbf{1}$ and $\bar{V}^{\dagger} \bar{V}=\mathbf{1}$ are restated as 


$$
\begin{gathered}
\sum_{n=1}^{N} \bar{p}_{n}=1, \\
\sum_{l=1}^{N} \bar{V}_{l n}^{*} \bar{V}_{l m}=\delta_{n m} .
\end{gathered}
$$

At this point, it is evident that the variational principles of maximum of entropy are the same in entanglement thermodynamics and statistical mechanics and that the principles of minimum of free energy are also the same in the two schemes. Hence, the principle of maximum of the entanglement entropy gives

$$
\left(C^{\dagger} C\right)_{n m}=\frac{1}{N} \delta_{n m}
$$

as the principle of maximum of entropy gives the microcanonical ensemble

$$
\tilde{P}_{n m}=\frac{1}{N} \delta_{n m}
$$

in statistical mechanics. Similarly, the principle of minimum of the entanglement free energy gives

$$
\left(C^{\dagger} C\right)_{n m}=Z^{-1} e^{-E_{n} / T_{e n t}} \delta_{n m}
$$

as the principle of minimum of the free energy gives the canonical ensemble

$$
\tilde{P}_{n m}=\bar{Z}^{-1} e^{-E_{n} / T} \delta_{n m}
$$

in statistical mechanics, where $Z=\sum_{n} e^{-E_{n} / T_{e n t}}$ and $\bar{Z}=\sum_{n} e^{-E_{n} / T}$. It is easy to see that (A12) and (A14) are equivalent to (9) and (16), respectively, up to a unitary transformation in $\mathcal{F}_{1}$.

Finally we comment on the generalization of the analysis when the Hilbert space is divided into two subspaces with different dimensions $\left(\operatorname{dim} \mathcal{F}_{1}>\operatorname{dim} \mathcal{F}_{2}\right)$. In this case, by defining $S_{\text {ent }}$ from $\rho_{2}$, we obtain similar results. 


\section{APPENDIX B:}

In this appendix we show that in the finite dimensional case the orthonormal basis $\left\{\left|\psi_{n m}\right\rangle_{1}\right\}$ defined in the physical principle (c) is given by (20) uniquely up to a unitary transformation in $\mathcal{F}_{1+}$. After that, we derive the equation (21).

We consider the following decomposition of the Hilbert space $\mathcal{F}_{1}$.

$$
\mathcal{F}_{1}=\mathcal{F}_{1+} \otimes \mathcal{F}_{1-}
$$

where $\mathcal{F}_{1+}$ and $\mathcal{F}_{1-}$ are Hilbert spaces with the same finite dimension $N$. From the arguments in Appendix A, each of the basis $\left\{\left|\psi_{n m}\right\rangle_{1}\right\}$ is obtained by applying a unitary transformation in $\mathcal{F}_{1+}$ to the following state in $\mathcal{F}_{1}$.

$$
|\phi\rangle_{1}=\frac{1}{\sqrt{N}} \sum_{j=1}^{N}\left(|j\rangle_{1+} \otimes|j\rangle_{1-}\right)
$$

where $|j\rangle_{1+}$ and $|j\rangle_{1-}(j=1,2, \cdots, N)$ are orthonormal bases of $\mathcal{F}_{1+}$ and $\mathcal{F}_{1-}$, respectively. Evidently, any states given by (20) are obtained by this procedure. Moreover, it is easily confirmed as follows that a set of all states given by (20) is a complete orthonormal basis in the $N \times N$ dimensional Hilbert space $\mathcal{F}_{1}$.

$$
{ }_{1}\left\langle\psi_{n m} \mid \psi_{n^{\prime} m^{\prime}}\right\rangle_{1}=\frac{1}{N} \sum_{j=1}^{N} e^{2 \pi i j\left(n^{\prime}-n\right) / N} \delta_{m m^{\prime}}=\delta_{n n^{\prime}} \delta_{m m^{\prime}}
$$

Let us suppose another complete orthonormal basis $\left\{\left|\bar{\psi}_{n m}\right\rangle_{1}\right\}$ in $\mathcal{F}_{1}$, each of which maximizes the entanglement entropy with respect to the decomposition $\mathcal{F}_{1}=\mathcal{F}_{1+} \otimes \mathcal{F}_{1-}$. Since both $\left\{\left|\psi_{n m}\right\rangle_{1}\right\}$ and $\left\{\left|\bar{\psi}_{n m}\right\rangle_{1}\right\}$ are complete orthonormal basis in $\mathcal{F}_{1}$, they are related by a unitary transformation $U$ in $\mathcal{F}_{1}$. Moreover, $U$ is a unitary transformation in $\mathcal{F}_{1+}$, since any states maximizing the entanglement entropy are related by unitary transformations in $\mathcal{F}_{1+}$

as shown in Appendix A. Therefore, the orthonormal basis $\left\{\left|\psi_{n m}\right\rangle_{1}\right\}$ defined in the physical principle (c) is unique up to a unitary transformation in $\mathcal{F}_{1-}$ and is given by (20).

Now let us show the equation (21). The right hand side is transformed as follows.

$$
\frac{1}{N} \sum_{n m}\left|\psi_{n m}\right\rangle_{1} \otimes U_{n m}^{(2+)}\left|\tilde{\phi}_{2}\right\rangle_{2}=\frac{1}{\sqrt{N}} \sum_{j k}\left(\frac{1}{N} \sum_{n} e^{2 \pi i(j-k) n / N}\right) \times \sum_{m m^{\prime} n^{\prime}} 2+\left\langle k \mid n^{\prime}\right\rangle_{2+} C_{n^{\prime} m^{\prime}}
$$




$$
\begin{aligned}
& \times|(j+m) \bmod N\rangle_{1+} \otimes|j\rangle_{1-} \otimes|(k+m) \bmod N\rangle_{2+} \otimes\left|m^{\prime}\right\rangle_{2-} \\
= & \sum_{m m^{\prime} n^{\prime}}\left(\frac{1}{\sqrt{N}}\left|\left(n^{\prime}+m\right) \bmod N\right\rangle_{1+} \otimes\left|\left(n^{\prime}+m\right) \bmod N\right\rangle_{2+}\right) \\
& \times C_{n^{\prime} m^{\prime}}\left|n^{\prime}\right\rangle_{1-} \otimes\left|m^{\prime}\right\rangle_{2-} \\
= & \left(\frac{1}{\sqrt{N}} \sum_{m^{\prime \prime}}\left|m^{\prime \prime}\right\rangle_{1+} \otimes\left|m^{\prime \prime}\right\rangle_{2+}\right) \\
& \times\left(\sum_{n^{\prime} m^{\prime}} C_{n^{\prime} m^{\prime}}\left|n^{\prime}\right\rangle_{1-} \otimes\left|m^{\prime}\right\rangle_{2-}\right) .
\end{aligned}
$$

The final expression is $|\phi\rangle$ itself. 


\section{REFERENCES}

[1] J. D. Bekenstein, Phys. Rev. D7, 2333 (1973).

[2] S. W. Hawking, Comm. math. Phys. 43, 199 (1975).

[3] V. P. Frolov and D. N. Page, Phys. Rev. Lett. 71, 3902 (1993).

[4] S. Mukohyama, Phys. Rev. D56, 2192 (1997).

[5] K. Maeda, T. Tachizawa and T. Torii, Phys. Rev. Lett. 72, 450 (1994).

[6] M. B. Green, J. H. Schwartz, E. Witten, Superstring theory (Cambridge University Press, 1987).

[7] J. Polchinski, S. Chaudhuri and C. V. Johnson, "Notes on D-Branes", hep-th/9602052 and references therein.

[8] J. M. Maldacena, "Black Holes in String Theory", hep-th/9607235 and references therein.

[9] J. H. Schwarz, in String Theory, Gauge Theory and Quantum Gravity, Proceedings of the Spring School, Trieste, Italy, 1996, edited by R. Dijkgraaf et al. [Nucl. Phys. B (Proc. Suppl.) 55B, 1 (1997)], hep-th/9607201, and references therein.

[10] T. Banks, W. Fischler, S. H. Shenker and L. Susskind, Phys. Rev. D55, 5112 (1997).

[11] M. Li and E. Martinec, Class. Quantum Grav. 14, 3187 (1997); 14, 3205 (1997).

[12] T. Banks, W. Fischler, I. R. Klebanov and L. Susskind, Phy. Rev. Lett. 80, 226 (1998); hep-th/9711005.

[13] C. Rovelli, "Loop Quantum Gravity", gr-qc/9710008 and references therein.

[14] A. Ashtekkar and K. Krasnov, "Quantum Geometry and Black Holes", gr-qc/9804039 and references therein.

[15] G. W. Gibbons, S. W. Hawking, Phys. Rev. D15, 2752 (1977); S. W. Hawking and G. 
T. Horowitz, Phys. Rev. D51, 4302 (1995); J. D. Brown and J. W. York, Jr., Phys. Rev. D47, 1420 (1993); M. Banados, C. Teitelboim and J. Zanelli, Phys. Rev. Lett. 72, 957 (1994).

[16] V. P. Frolov, D. V. Fursaev and A. I. Zelnikov, Phys. Rev. D54, 2711 (1996).

[17] R. M. Wald, Phys. Rev. D48, R3427 (1993).

[18] V. Iyer and R. M. Wald, Phys. Rev. D52, 4430 (1995).

[19] G. 't Hooft, Nucl. Phys. B256, 727 (1985).

[20] F. Pretorius, D. Vollick, and W. Israel, Phys. Rev. D57, (1998).

[21] L. Bombelli, R. K. Koul, J. Lee and R. D. Sorkin, Phys. Rev. D34, 373 (1986).

[22] M. Srednicki, Phys. Rev. Lett. 71, 666 (1993).

[23] For example, J. D. Bekenstein, gr-qc/9409015; E. Benedict and S. Pi, Ann. Phys. 245, 209 (1996); C. Callan and F. Wilczec, Phys. Lett. B333, 55 (1995); S. R. Das, Phys. Rev. D51, 6901 (1995); J. S. Dowker, Class. Quantum. Grav. 11, L55 (1994); V. Frolov and I. Novikov, Phys. Rev. D48, 4545 (1993); C. Hozhey, F. Larsen and F. Wilczek, Nucl. Phys. B424, 443 (1994); D. Kabat, Nucl. Phys. B453, 281 (1995); F. Larsen and F. Wilczek, Ann. Phys. 243, 280 (1995); S. Liberati, Nuov. Cim. 112B, 405 (1997); R. Muller and C. Lousto, D52, 4512 (1996); S. Solodukhin, Phys. Rev. D54, 3900(1996).

[24] S. Mukohyama, M. Seriu and H. Kodama, Phys. Rev. D55, 7666. (1997).

[25] S. Mukohyama, M. Seriu and H. Kodama, gr-qc/9712018, to appear in Phys. Rev. D.

[26] N. J. Cerf, and C. Adami, Phys. Rev. Lett. 79, 5194 (1997).

[27] N. J. Cerf, and C. Adami, Phys. Rev. A55, 3371 (1997).

[28] For example, G. Jumarie, Relative information (Springer, 1990).

[29] C. H. Bennet, G. Brassard, C. Crépeau, R. Jozsa, A. Peres, and W. K. Wootters, Phys. 
Rev. Lett. 70, 1895 (1993).

[30] D. Bouwrester, J-W. Pan, K. Mattle, M Eibl, H. Weinfurter, and A. Zeilinger, Nature 390, 575 (1997).

[31] D. Boschi, S. Branca, F. De Martini, L. Hardy, and S. Popescu, Phys. Rev. Lett. 80, 1121 (1998).

[32] W. Israel, Phys. Lett. 57A, 107 (1976).

[33] L. Parker, Phys. Rev. D12, 1519 (1975).

[34] V. Frolov and I. Novikov, Phys. Rev. D48, 1607 (1993).

[35] L. Susskind and J. Uglum, Phys. Rev. D50, 2700 (1994).

[36] V. Frolov, D. Fursaev and A. Zelnikov, Nucl. Phys. B486, 339 (1996).

[37] S. Mukohyama and W. Israel, gr-qc/9806012, to appear in Phys. Rev. D. 